\title{
NUEVAS MASCULINIDADES Y NARRATIVAS DE EXCOMBATIENTES DE LAS FAR DESPUÉS DEL POSACUERDO DE LA HABANA
}

\author{
NEW MASCULINITIES AND NARRATIVES FROM EX FARC MEMBERS \\ AFTER THE HAVANA PEACE AGREEMENT
}
NOVAS MASCULINIDADES E NARRATIVAS DOS EX-COMBATENTES DAS FAR APÓS O ACORDO DE HAVANA

\author{
Brian Camelo Niño \\ Universidad Nacional de Colombia, Colombia \\ https://orcid.org/0000-0003-1037-2513 \\ bacamelon@unal.edu.co
}

Recibido: 29 /07/2020 Revisado:13/08 /2020 Aceptado:14/08/2020 Publicado: 01/11/2020

Resumen: Este articulo recoge la investigación sobre las relaciones de las nuevas masculinidades y la paz en el escenario de posacuerdo de La Habana con las FARC. El método de investigación fue, en primera instancia un análisis documental y, después, una serie de siete entrevistas semiestructuradas con hombres y mujeres activistas en la temática de nuevas masculinidades. En las narrativas que presentaron los y las activistas se encontraron aspectos discursivos en común, como masculinidades no hegemónicas, y dos categorías "criollas", libertarias e insurgentes, además, de su visión sobre la paz y la implementación del acuerdo firmado en La Habana. A partir de la teoría de la paz imperfecta, se buscó resaltar la experiencia y los avances más importantes que tiene la perspectiva relacional de género en la construcción de escenarios de convivencia más pacíficos. Así mismo, fue explorada la creación de marcos discursivos para la construcción de paz en Colombia.

Palabras claves: Paz; Masculinidades; Narratividad; Posacuerdo de La Habana;FARC.

\begin{abstract}
This article gathers information about the relationship between new masculinities and peace in Colombia after the signing of the Havana Peace Agreement. The investigation started with a literature review, followed by a series of semi-structured interviews with both female and male activists of new masculinities. Based on the imperfect peace theory, the focus of this paper is on the experience and advances of the relational perspective of gender in the construction of peaceful coexistence scenarios. There were various common aspects among the activists' narratives including non-hegemonic masculinity, two categories of creoles, libertarians and insurgents, as well as their vision about peace and the implementation of the agreement signed in Havana. In the same way, the creation of discursive frameworks for peacebuilding in Colombia was explored.
\end{abstract}

Keywords: Peace; Masculinity; Narratives; Post Havana Peace Agreement; FARC

Resumo: Este artigo relata a pesquisa sobre as relações entre as novas masculinidades e a paz no cenário seguinte ao acordo de Havana con as FARC. O método de pesquisa foi uma análise documental e uma série de sete entrevistas semi estruturadas com homens e mulheres ativistas do tema de novas masculinidades. A partir da teoria da paz imperfeita se buscou enfatizar a experiencia e os avances mais importantes da perspectiva relacional de género na construção de cenários de convivencia mais pacíficos. Nas narrativas que se apresentaram, as(os) ativistas tiveram aspectos discursivos em comum, como as masculinidades não hegemónicas, e duas categorías locais, libertarias e insurgentes, assim como sua visão sobre a paz e a implementação 
do acordo assinado em Havana. Da mesma forma, foi explorada a criação de marcos discursivos para a construção da paz na Colômbia.

Palavras-chave: Paz; Masculinidades; Narrativa; Acordó de Havana; FARC.

Cómo citar este artículo: Camelo, B. (2020). Nuevas masculinidades y narrativas de excombatientes de la Far después del posacuerdo de La Habana. Hachetetepé. Revista científica en Educación y Comunicación, (21), 44-55. https://doi.org/10.25267/Hachetetepe.2020.i21.5

\section{INTRODUCCIÓN}

Este artículo es un acercamiento a las masculinidades no hegemónicas en el contexto colombiano de posacuerdo con la guerrilla de las FARC en La Habana y sus luchas por construir la paz. Así mismo, es un intento por descifrar las nuevas formas de ser hombre o, en su defecto, retirarse de la esfera de privilegios en la que se ubica al varón en el ordén hetero patriarcal. No sólo se pretende construir conocimiento desde los distintos caminos que han sido recorridos por activistas en la lucha por la igualdad de género, es también, en sí mismo, un ejercicio por acercarse al lado más humano de la emocionalidad y afecto en las ciencias sociales.

En medio de múltiples escenarios, se desarrollan masculinidades que están en consonancia con juicios éticos y morales establecidos desde las dinámicas sociales propias de cada contexto. El sistema patriarcal impone una serie de valores que están en conformidad con el sistema económico capitalista y neoliberal "ser hombre es, de entrada, hallarse ante una posición que implica poder"(Bourdieu, 1996, p.21). En medio de una matriz que involucran el capital monetario, el capital simbólico, el capital erótico, valores como la justicia social, la igualdad de los géneros, el derecho a cuidar de los otros, quedan en un segundo plano.

En este sentido, la firma de la paz en Colombia y su búsqueda se vio revitalizada con el impulso del género y el papel de las mujeres. En el acuerdo de paz que se firmó en La Habana se presentaron varios aspectos excepcionales dentro de los cuales cabe señalar el papel activo de las mujeres para "establecer" el enfoque de género como un elemento indispensable para realizar el acuerdo de paz, siendo pionero, no solo en la historia de Colombia sino a nivel mundial.

Uno de los aspectos más interesantes del acuerdo de paz en La Habana es como hoja de ruta para consolidar una paz estructural y simbólica en Colombia, que se inicia con un análisis histórico de las condiciones que han favorecido la génesis, mutación y perpetuación de la violencia durante los dos siglos de vida republicana que lleva el país. Según el profesor Moncayo (Estrada y Jiménez, 2019) para hacer un análisis del proceso de paz en Colombia es necesario primero estudiar el modelo económico en el que se consolidó el conflicto armado, que es el capitalismo a partir de la acumulación de la tierra en pocas manos, con un proceso de distribución de la riqueza profundamente desigual y el surgimiento de dos bloques, el insurgente y el contrainsurgente. Con la firma del pacto de La Habana se buscó generar un nuevo orden social que contribuyera a cerrar las brechas que existían dentro de la población en cuanto a ingresos y oportunidades de desarrollo en el ámbito rural y urbano. La paz a la que le apostaba el gobierno de Juan Manuel Santos en sus inicios era excesivamente básica o sin garantías para las FARC, una paz negativa que sólo implicaba el desarme, la desmovilización y la reintegración de la insurgencia en la vida civil (Estrada y Jiménez, 2019). Sin embargo, con el acuerdo base de los seis puntos que se firmó y los tres ejes transversales se logró entregar al país

e-ISSN:2172-7910

Doi:10.25267/Hachetetepe.2020.i21.5

Universidad de Cádiz 
una hoja de ruta que permitía salir de las condiciones sociales del atraso que lo habían llevado a la guerra y se daban las garantías políticas a los y las excombatientes de participar en la creación de un orden social nuevo.

El enfoque de género fue un avance que tuvieron que dar las mujeres, la academia y representantes del gobierno que se construyó a partir de la experiencia de la vida guerrillera y el dialogo con sectores del gobierno y organismos internacionales como ONU Mujeres. El punto sobre género fue uno de los aspectos álgidos del acuerdo, debido a la reacción en contra por parte de los sectores más conservadores y su alianza con grupos religiosos del escenario de la derecha en Colombia que lograron, incluso, que en una votación histórica no se ratificara por la vía de las urnas. Finalmente, se logró salir adelante, reconociendo la necesidad de su trasversalidad.

Inmersos en la temática del género, está el papel de los varones en la construcción de un escenario más equitativo en cuanto a funciones y roles sociales que se deben desempeñar en los nuevos escenarios de la vida civil. Se parte de la premisa de las masculinidades no hegemónicas como aspecto esencial para construir relaciones de convivencia comunitarias que ayuden a construir una paz real.

\subsection{La paz y su relación con el género}

Comprendemos que el abordaje de la paz debe ser desde un campo transdisciplinar que se abre a todas las materias que puedan contribuir de forma significativa a su interpretación. Así mismo, creemos que a partir de las entrevistas con personas que hayan abordado la temática podemos hacer que sea expuesta desde la praxis:

La paz participa de lo real, pero ella misma se superpone a lo real; participa del sujeto, pero determina al sujeto y es éste quien aplica o la disfruta. Está reflejada en el lenguaje y es constituida por el lenguaje. Es una institución cultural y las culturas la instituyen y destituyen. Y, en consecuencia, puede y debe ser mirada desde una «praxis» que toma dimensiones prácticas reelaboradas desde la internalización emocional, cognoscitiva y teórica. (Muñoz, 2001, p.31)

De esta manera, el concepto de paz imperfecta se muestra fecundo para abordar el proceso de paz en Colombia por el reconocimiento de la conflictividad como un proceso inherente de las relaciones humanas. Ya, en la década de 1980, Zuleta (1991), siguiendo a Kant, decía desde Colombia que sólo un pueblo escéptico con la fiesta de la guerra y maduro para el conflicto merece la paz. Desde esta visión, el conflicto es inherente a las relaciones humanas, siendo, lo que diferencia a una sociedad de las demás, la forma en la que sus ciudadanos dirimen sus diferencias, por la violencia o por medio del dialogo y otros elementos.

La paz imperfecta se apoya en cuatro aspectos que es importante resaltar: Primero, es alcanzable, no tiene que ser algo ideal, alejada, o tiene que cumplir con unos requisitos esenciales (idílicos); segundo, nos ayuda a visibilizar pequeñas experiencias de paz que construyen una paz mayor; tercero, nos ayuda a planificar futuros donde la imperfección de los seres humanos, sus pasiones y sus conflictos tienen cabida; cuarto, su contenido prospectivo nos posiciona para ver los posibles escenarios futuros que puede tener la paz imperfecta.

Igualmente, el acuerdo de paz de La Habana es pionero en la historia, en cuanto es el primero en el que se logró implementar de forma exitosa el enfoque de género en las 130 medidas afirmativas que contempló para promover la igualdad de derechos de hombres y mujeres. Para que se logrará el enfoque transversal de género en el acuerdo de 
paz de La Habana fue necesario que existiera una matriz internacional de leyes y protocolos que surgió desde la década de 1990 en las inquietudes que planteó la declaración y plataforma de Beijing, la declaración de Windhoek y, finalmente, las directrices establecidas por el Consejo de Seguridad del ONU en la resolución 1325 del 2000. Además, del compromiso político de toda la comunidad internacional y avances propios que habían realizado las FARC en cuanto al género.

\subsection{Masculinidades y violencia en Colombia}

La masculinidad y la violencia en Colombia se mueven en múltiples tensiones: educación y cultura, la violencia pública y la privada, la territorialización de la guerra en el cuerpo, violencia sexual, etc. De las casi 25000 víctimas que existen de la violencia sexual, la abrumadora mayoría son mujeres, lo que nos lleva a preguntarnos ¿Por qué la violencia es ejercida mayoritariamente por hombres? Para iluminar el debate, Raewyn Connell (2013), nos ofrece las siguientes variables que contribuyen a fomentar un ambiente masculino tóxico:

1. El desprecio social de las mujeres. Las culturas patriarcales definen a los hombres como más importantes que las mujeres, y enseñan visiones estereotipadas acerca de para qué sirven y cómo deberían comportarse las mujeres. Existen profundas raíces históricas de misoginia en el colonialismo y la religión, pero algunas son bastante actuales, de lo cual es un ejemplo la pornografía.

2. La hegemonía de una forma de masculinidad que enfatiza el poder y la dominación, y un sentido entre hombres de un derecho a ejercer su poder sin restricción, dentro de la familia o fuera de ella.

3. Un ambiente que respalda la violencia de género. Esto incluye los medios de comunicación saturados con violencia, el apoyo entre pares a la violencia entre hombres y a la impunidad frente a las acciones violentas. (p. 263)

De la misma forma, Millán, et al. (2015) realizaron algunos talleres en la zona de Buenaventura, encontrando hallazgos significativos que nos pueden ayudar a comprender la dimensión cultural y la relación de las masculinidades mal llevadas con la violencia en un contexto de abandono estatal. Las mujeres que participaban en el taller mostraban inquietud respecto a los juegos en los que participaban sus hijos, en los que era común que los niños jugaran a perseguir a las niñas con un palo, que simula ser su pene y las niñas corren, para evitar las violaciones. Otro juego común, era el de "ser propietarios de casas de pique" que es donde descuartizan a las personas después de asesinarlas, para hacer desaparecer su cuerpo. A través de juegos y lecturas que hacen de su compleja realidad los sectores más desfavorecidos de la sociedad colombiana empiezan a cultivar una subjetividad masculina guerrera que a su vez genera feminidades cosificadas en el complejo mundo de las relaciones de género en una economía de guerra:

A los 12 años me gustaba llegar de la jornada de trabajo y ser parte de alguna de las bandas que teníamos con mis amigos: hacíamos pistolas con palos y caucheras, nos vengábamos de los que considerábamos nuestros enemigos $\mathrm{y}$, a veces, dejábamos amarrado en un árbol a algún niño que nos cayera mal. Era un juego. Eso pensábamos, hasta que los 'paras' nos vieron e intentaron reclutarnos. (Rubio, 2013, p.21)

Las economías de guerra que se han consolidado en lugares donde existe escasa presencia del Estado ayudan a que los niños y niñas vean las armas, los uniformes y la 
violencia como símbolo de prestigio y única manera de lograr escalar socialmente. La cultura queda relegada a los símbolos de prestigio de la guerra. En los grupos paramilitares un ejemplo fue la "cosificación" y el "prestigio" que daba tener muchas mujeres, como lo expuso un excombatiente de las Autodefensas Unidas de Colombia; "En este país tener un arma es ser un hombre con poder, poder salir con las mujeres más "bonitas" y vestirse bien" (Theydon, 2009, p.13).

Las masculinidades en la guerra forjan nuevas formas de performatividad, como las exploradas por la antropóloga Theydon Kimberli (2009) en las caras de los combatientes, la máscara del guerrero, que implican posturas de tensión que generan en las relaciones sociales con los otros; miedo, odio, etc. Estas formas de performatividad operan en todo el entramado social, y esto se evidencia en las narraciones que realizó la antropóloga de sus encuentros con hombres pertenecientes a grupos paramilitares, desde el trato que les daban en la recepción del hotel, hasta la forma en la que los miraban, que es una mezcla de miedo y prestigio.

Dentro de las masculinidades hegemónicas, Muñoz-Onofre (2011, p.92) creó la categoría analítica de masculinidades bélicas, para entender cuál es el contexto que facilitó la aparición de este conjunto cultural y de prácticas en los varones colombianos:

- Cohabitación de las comunidades rurales con los grupos armados irregulares.

- Presencia normalizada de la figura modélica del combatiente armado (sea este soldado regular, paramilitar o guerrillero).

- Juegos bélicos practicados durante la infancia.

- Obligación de desempeñar labores agrícolas pesadas en edades tempranas.

- Maltrato recibido en la familia, entre las más importantes.

Si bien, este es el caldo de cultivo para las masculinidades bélicas, también se van consolidando en relación con una serie de valores que son importantes durante el adiestramiento militar: Aguante físico, endurecimiento emocional, normalización de la muerte, pruebas de fidelidad al grupo, indiferencia frente al sufrimiento de otros, capacidad de matar, etc. Este conjunto de valores se ven replicados en todos los ejércitos que se involucraron en la guerra en Colombia; Fuerzas armadas, Paramilitares, Guerrillas y cuerpos privados de seguridad.

Finalmente, todo lo anterior, nos lleva a determinar que las masculinidades guerreras no sólo están presentes en los combates armados, sino, también en la cotidianidad y la cultura. Las masculinidades toxicas atraviesan desde los juegos de los niños, hasta la performatividad de los combatientes, pasando por la apropiación y reproducción cultural que se da en las poblaciones más desfavorecidas y alejadas del centro económico y político de Colombia.

En el otro lado del espectro, se encuentran con un menor uso de los medios de comunicación y menos visibilidad las masculinidades disidentes o alternativas. En este momento en Colombia existen más de treinta iniciativas colectivas que intentan cambiar la forma de ser hombres desde una matriz de valores totalmente opuestos a las masculinidades hegemónicas guerreristas que se han impulsado históricamente desde el statu quo colombiano.

En el ámbito de las intervenciones sociales y políticas, se encuentra el Colectivo Hombres y Masculinidades, que de manera ininterrumpida desde 1994, adelanta labores en distintas partes del país, junto a otras veintiséis iniciativas. Entre ellas están el Grupo de Nuevas Identidades Masculinas del Suroeste Antioqueño, el 
Grupo de Hombres de Casitas Bíblicas / Bogotá, el Colectivo (juvenil) Sin Fronteras/ Bogotá, los grupos de TransMasculinidades / Bogotá, el Equipo Masculinidades Caribe, el Círculo de Hombres y Taller Abierto/ Cali, Hombres en Marcha /Pasto, Machos Afectivos /Manizales, y Grupo Combos/ Medellín. Para ir articulando estas experiencias, los distintos grupos y personas activistas han confluido en espacios como la Red Colombiana de Masculinidades por la Equidad de Género, la Red Colombiana de Masculinidades No Hegemónicas, y la Mesa Nacional de Masculinidades Corresponsables y No Violentas (Arroyave, 2018, p.2)

Estas iniciativas tienen como puntos en común sus posturas criticas ante el sistema capitalista, patriarcal y colonial. Dentro de estas otras formas de ser hombre mediadas por diversos adjetivos que encierran las características están; Nuevas Masculinidades, No Hegemónicas, No Violentas, Masculinidades Corresponsables, Alternativas, Liberadoras, Libertarias, y una categoría relativamente nueva que es la de insurgentes.

\section{METODOLOGÍA}

Para la elaboración de este trabajo elegimos, la investigación cualitativa. Consideramos que el conocimiento del entorno social y las circunstancias históricas que lo han configurado son factores decisivos en la configuración de prácticas nuevas y alternativas culturales que desemboquen en una sociedad más justa en términos estructurales. Así mismo, los investigadores y los investigados a partir de la interacción descubren nuevos escenarios de interpretación, liberación y transformación social.

Se eligió la entrevista semiestructurada como técnica de recolección de la información debido a que nutre la reflexión a partir de las experiencias de los y las activistas en nuevas masculinidades. Es un método que ayuda en la comprensión de los mundos de la vida que han ido tejiendo los hombres y mujeres que participan activamente en los debates sobre paz, género y política.

Además, permite la interacción del investigador con los y las investigadas: "este tipo de entrevista facilita la recolección y el análisis de saberes sociales cristalizados en discursos, que han sido construidos por la práctica directa y no mediada de los protagonistas" (Tonón,2008, p. 50). El hecho de elaborar una serie de preguntas abiertas permite al investigador una mayor flexibilidad en la profundización de acuerdo con el dialogo que se vaya tejiendo y las inquietudes de los entrevistados. El mundo de la vida de los entrevistados es puesto en común a partir de inquietudes que generan una revisión biográfica. Se ponen en juego, entonces, los universos culturales y experiencias de los dos sujetos que se ven e intercambian conocimientos.

El ambiente abierto de la entrevista semiestructurada facilita que los sujetos de la investigación exploren líneas de comprensión de la realidad que el investigador no había tenido en cuenta. Además, "es una técnica que facilita la libre manifestación de los sujetos de sus intereses informativos (recuerdo espontáneo), sus creencias (expectativas y orientaciones de valor sobre las informaciones recibidas) y sus deseos" (op cit, p.53) todo esto en un ambiente de información que no sólo incluye la oralidad, sino, también la proxémica, la entonación, e incluso los silencios.

En la elaboración de la entrevista semiestructurada se tomaron cuatro categorías que se expusieron en las cuestiones de la investigación: Concepto de la masculinidad, relación con la política, posibilidades de transformación y relación con la paz. 


\section{RESULTADOS}

En los aspectos en común encontramos que para todas las entrevistadas y entrevistados las masculinidades son construcciones culturales, alejándose de la visión biológica de la sexualidad. Una categoría que es abordada en todos los discursos es la de masculinidades hegemónicas o tóxicas, excepción realizada por el grupo que trabaja en las FARC que tienen en paralelo un discurso sobre la masculinidad de la vida civil, en contraposición, a la que llevaban en la vida guerrillera y la que encontraron después del proceso de paz cuando se reintegraron, que, a pesar de no ser teorizada, se acerca mucho a la teoría de Connell:

Desde la insurgencia esos logros que habíamos tenido en igualdad entre hombres y mujeres empezamos a verlos esfumarse con la reincorporación y con el proceso de paz paradójicamente, eh, empezamos a retroceder entonces era una cuestión como más interna y como más de ver lo que estábamos perdiendo. (Víctor, militante partido FARC, comunicación personal, 17 enero 2020).

Desde el proceso de reincorporación se presentan temas delicados en el asunto de género. Algunas de nuestras compañeras comenzaron a caer de nuevo en los estereotipos de género, entonces las mujeres que eran políticamente muy activas empezaron a estar en la casa, con el cuidado de los niños, lavado de ropa, ese tipo de cosas y los manes (hombres) ya no querían hacer nada. En la guerrilla la distribución de tareas era muy equitativa. Hubo escenarios de violencia intrafamiliar, también, cosas que antes no se daban dentro de la organización porque existían unas normas en torno a eso y eran estrictas. Entonces los manes comenzaron a decir que las nenas (mujeres) que trabajábamos en género les lavábamos la cabeza.

(Silencio)

Entonces decidimos que, que, si no nos dejaban entrar, teníamos que empezar a realizar un trabajo con los hombres. (Angélica, Militante partido FARC, comunicación personal, 17 de enero del 2020)

En el contexto colombiano, el tema la masculinidad hegemónica ha permeado todos los escenarios de socialización. El discurso se vive en grados distintos, dependiendo del contexto en el que se desenvuelvan los sujetos, campesinos o urbanos, pero, existen tópicos comunes que determinan que los hombres entren en dinámicas de poder vertical mediadas por su sexo, existe una ritualidad asociada a la dominación masculina, Bourdieu (1996).

Cuando se produce la desmovilización de las FAR en el espacio de la vida civil ya hay una subcomisión de género, y la inquietud en torno a como se va a hacer el trabajo en la sociedad civil. Por eso, en el momento en el que se detectan las violencias de género, la vinculación de las mujeres solo al trabajo doméstico, y su relegación a la esfera de lo privado existe una respuesta por parte del partido que es la implementación de la subcomisión de nuevas masculinidades.

Siguiendo a Muñoz y Jiménez (2009), se podría hablar acerca de la existencia de una paz imperfecta de género en la cultura guerrillera, previa a la firma del acuerdo de paz en La Habana, en sus palabras:

Es hablar en primer lugar, de todas las instancias de paz, por muy pequeñas y aisladas que sean o estén, de las que forma parte el género, sean las protagonistas las mujeres, los hombres o las relaciones entre ellos. La paz imperfecta de género es imprescindible para reconstruir el poder, el empoderamiento, de las mujeres" (p.424). 
Las mujeres farianas (militantes femeninas de las FARC) tenían elementos de empoderación, en las labores realizadas durante su periodo activo en las FARC EP, es decir, se habían descubierto a sí mismas como actoras políticas, no simples entes pasivos. $\mathrm{Su}$ papel como guerreras también contribuyó a general un rol activo dentro de la organización que no se encontraba restringido a la economía doméstica del cuidado.

No sobra decir que en el contexto de la guerra se presentó también violencia de género por parte las FARC EP. Sin embargo, en la práctica, para los y las entrevistadas se hizo indispensable ver el género desde una postura relacional critica con los valores hegemónicos construido en la sociedad civil.

En ese sentido, la masculinidad es vista por los y las entrevistados como una categoría que se desarrolla en las interacciones que tiene el varón con su entorno. Siendo la primera, la hegemónica, que se ancla en una estructura de valores sociales que va en consonancia con el estilo de vida en el que se desenvuelven, en el caso de la sociedad civil a la que llegaron después del proceso de paz de La Habana, individualista, capitalista, en oposición, al comunitario guerrillero que llevaban.

Con relación a las fuentes particulares, que nos brindaron nuestros y nuestras entrevistados, es necesario examinar que no todos tienen que ver directamente con el trabajo de masculinidades, sino, que provienen de otras vertientes, de las cuales se nutren: Michael Kaufman, Wilhelm Reich, Alexander Lowen, Rolando Toro, Alejandro jodorowsky, Rodolfo kush, Carlos Ivan García, Manuel Roberto Escolar, José Fernando Serrano, Rita Segato.

Así mismo, las nuevas masculinidades en Colombia han desarrollado un proceso de convergencia, donde se dan citas, los autores latinoamericanos de la filosofía de la liberación, el teatro del oprimido, la psicomagia, la biodanza, las teorías indígenas del buen vivir en la categoría de masculinidades libertarias o liberadoras. En el mismo sentido, se encuentran las experiencias de la milicia en la guerrilla, donde los hombres pasan de ser insurgentes en armas a serlo en la sociedad civil, en la medida en que ven un modelo económico opresor con mujeres y hombres. En cuanto a los adjetivos de las masculinidades estudiadas, hay dos que son particularmente relevantes para nuestro estudio, liberadoras o libertarias e insurgentes.

En este orden de ideas, después de la implementación del acuerdo de paz en las entidades territoriales se estaba dando un proceso de enajenación en los excombatientes que olvidaron las prácticas igualitarias de la vida comunitaria lo que llevó a la comisión de género a pensar en una nueva masculinidad que acompañara al feminismo insurgente en su lucha por una sociedad distinta, al menos en los Espacios territoriales de capacitación y reincorporación (ETCR).

Entonces empezamos a formar a los chinos, bueno, viejos (risas) y de ahí surgió un grupo base de veinte manes. Ellos van a hacer parte del primer encuentro nacional de masculinidades insurgentes. De ese encuentro surgió también, un plan de trabajo. Entonces la idea es que en cada ETCR se trabaje en género y masculinidad insurgente. (Angelica, militante partido Farc, comunicación personal, 15 de enero del 2020)

En este sentido, consideramos que ser activista en masculinidades nuevas en Colombia pasa por la conciencia del cuerpo como territorio, su "relacionalidad" con la familia, el padre, la madre y la sociedad. En un segundo momento, se encuentra su "emocionar", el desear, sentir, odiar, querer, amar nuevas formas de ser y existir como 
hombres y mujeres. Y el último estadio, que sería el de racionalizar, entendido como la capacidad de pensar por sí mismo, ponerse en el lugar del otro u otra y ser consecuente con los ideales que se traducen en el deber político de participar en la construcción de nuevas relaciones, palabras, contextos en el campo de la cultura, transformando y tocando las relaciones de poder.

De la misma forma, La política y lo político son escenarios que permean el discurso de todos los y las activistas en masculinidades. La categoría de "urgente" se impone a la de "importante" en varios de los discursos estudiados, además, de la preocupación por la lucha contra el patriarcado. Así mismo, no se ven las nuevas masculinidades como confrontativas con el discurso feminista, incluso, dos de las entrevistadas se mueven en la frontera del género en todas sus variantes y las políticas con relación a la sexualidad.

Yo creo que es absolutamente importante, necesaria y urgente, sobre todo digamos las acciones deliberadas que permitan replantear las lógicas masculinas, instaladas en la cultura, en la academia, en la política, en la vida doméstica. Sí, es importante y por qué razón es importante porque al analizar y al ver las maneras cómo operan las lógicas de género en sociedades como las nuestras encontramos que definitivamente allí ópera una lógica masculina hegemónica dominante patriarcalizada. (Javier, Hombres y masculinidades, comunicación personal, 11 de mayo del 2020)

La categoría de análisis de masculinidad insurgente donde nos ubicamos nosotros parte de una razón principal: uno, identificar el patriarcado como sistema de dominación, sí, e identificar la masculinidad como una de las culturas que sirve de herramienta para establecer y darle continuidad a ese mismo sistema patriarcal. Eh, que igual forma como patriarcado también sirve para dar legitimidad y seguir estableciendo el estatus quo del capitalismo, sí, entonces en esas razones nosotros creemos que plantearnos o repensarnos nuestra masculinidad desde una motivación insurgente hace que se piensen los cambios y las transformaciones sociales, entonces eso lo hace político. (Alejandro, activista partido FARC)

En los y las activistas se evidenció que el acuerdo de paz de La Habana fue un avance a nivel jurídico, pero, lo que se buscaban en realidad, es una paz, de tipo más estructural, que implique un dialogo y un desarrollo con sectores campesinos, con lo más desfavorecidos en el territorio nacional a partir de elementos como la justicia social.

Creo que tenemos que trabajar muy intensamente, esta apuesta es muy grande, tiene resonancias y tiene implicaciones sociales y políticas muy grandes ante un Estado y una clase política como la que tenemos que sabemos que no cuenta con la voluntad política del adelantar proyecto de transformación social significativa. (Javier, activista, Hombres y masculinidades, comunicación personal, 11 de mayo de 2020)

Así mismo, en el siguiente relato de Alejandro, sobre qué considera la paz, se tejen la sensación interior, el reencuentro con lo social, y la necesidad de una paz estructural entendida como potencialidad de transformación de las condiciones sociales para el desarrollo. Hay una visión interna que se expande al reencuentro con los otros y finaliza en un análisis de las condiciones estructurales que se necesitan para que se dé la paz real en los territorios:

Ayer yo estaba en el Guaviare y tuvimos un tiempo y nos fuimos al río, a los caños a descansar, a pasear, y el vuelo de nosotros era como a las 4 de la tarde y era ya 
como las 2 .Y yo estaba todavía en el caño metido y me decía la compañera que pues rápido, que nos fuéramos porque íbamos a perder el vuelo, porque los afanes y entonces, pues yo estaba, pues ahí metido viendo los pescaditos, con las patas ahí entretenido, viendo, pendejeando, entonces yo me asomé, yo saqué la cabeza y le dije no, no, compañera a nosotros también nos prometieron la paz y para mí esto es paz, estar aquí tranquilo sí, entonces con esa analogía tan sencilla de que primero a nosotros desde la clandestinidad también se nos prometió una paz que está llegando de otras maneras, no con el proceso de reincorporación, sino con la forma de vernos con el hermano, vernos con la hermana con toda la insurgencia que estuvo peleando en el monte por nosotros por Colombia, ese abrazo fraterno de la paz sí?, ese volvernos a encontrar, y nos encontramos cada día con gente que uno hace muchos años no veía y eso es alegría y eso es paz, es la paz del silencio de los fusiles y de la guerra, sí? que qué quisiera yo que fuera la paz? pues mayor amplitud y oportunidades para la gente, para el campesinado colombiano, oportunidades de progreso por proyectos productivos que permitan pensarse de forma diversa y crítica la participación de hombres y mujeres e infancias en la construcción de esos territorios de paz pero pues ahí para allá es más complicado. (Alejandro, militante, partido FARC)

\section{DISCUSIONES Y CONCLUSIONES}

Los principales discursos que se evidenciaron en los y las activistas transitan por un primer acercamiento con la masculinidad hegemónica Connell (2003), ya sea por el contexto de crianza, el vínculo con el padre, o por las dinámicas de relación cultural que se producen cuando los excombatientes se reintegran a la sociedad civil, y vuelven a ver como algunas de sus compañeros y compañeras caen en los mismos roles y estereotipos de género, alejados de las dinámicas de poder que se pretendían horizontales dentro de la organización, antes de la firma del acuerdo de paz en La Habana.

Al respecto, podríamos hablar de un contexto masculinizante (Méndez, 2002) que es necesario modificar, y de hecho es modificado a partir de la introducción de nuevas palabras en el discurso, como, por ejemplo, masculinidades insurgentes y libertarias o liberadoras y valores asociados a ellas; la paternidad responsable, la insurgencia contra el orden establecido, la liberación del cuerpo, a través del des-nudo, la palabra, la ternura, la vivencia de la sexualidad, el respeto, el sentipensamiento etc., que se construyen como nuevos marcos que contribuyen en la construcción de nuevas formas de ser hombre y contribuir a la paz, desde la esfera de lo intrapersonal hasta lo público.

En cuanto a las relaciones que tienen las masculinidades no hegemónicas con la paz, se pueden establecer una simbiosis, no se puede pensar la paz sin incluir las dinámicas de género y las relaciones de los sexos en la esfera de lo privado y lo público. La paz es una categoría polisémica, relacional, con una capacidad infinita de transformarse y transformar la sociedad. La paz imperfecta que se vive en los espacios territoriales de los firmantes de las FARC es un espacio de construcción único, en el que se pueden transmutar la vivencia de los conflictos como oportunidades de avance social, algunos ejemplos de ellos son: la comisión de género del partido, las campañas y actividades políticas que se han venido trabajando desde el feminismo y la masculinidad insurgente.

En el futuro, esperamos, que al menos en los espacios territoriales de capacitación y reincorporación (ETCR) de las FARC se pueda hablar de nuevas formas de ser hombre, 
que el primer acercamiento de los niños no se haga a través de la masculinidad tóxica, sino a través de otras formas de ser y estar en el mundo.

Estas nuevas masculinidades tienen que surgir de la mimesis y de forma centrípeta, es decir, mediante el cambio que hagan los adultos, la imitación que hagan los más pequeños y la reproducción cultural como elemento valioso y viable en la sociedad. Lo más importante, en este sentido, que dejó la firma del acuerdo de paz de La Habana es que se empezaron a ver valores morales nuevos para las relaciones de género, que en el plano de las palabras se empezó a discutir con fuerza en los territorios sobre la equidad de género.

Por otro lado, los retos a los que se enfrentan las nuevas masculinidades son muy grandes, ya que implican la construcción de una sociedad totalmente distinta a la que es imperante, ahora. Pensar en lógicas no patriarcales, también, es abandonar la violencia en el lenguaje, la beligerancia. Todo el tiempo estamos inmersos en el discurso de las luchas, de la hegemonía, del combate, la pregunta que queremos dejar en el aire es: ¿y si nos empezamos a pensar en discursos más cooperativos, en la no necesidad de disputar el poder, sino de crear lazos de solidaridad, de amor y afectividad? Suena idílico, pero, es viable, como lo demostró Muñoz (2001) en la naturaleza humana esta también la paz, la cooperación, más allá de la violencia. Noha (2014) también nos mostró como las sociedades humanas hemos logrado avanzar en el tiempo gracias a las redes y la flexibilidad colaborativa, además, del cotilleo. En consecuencia, sería interesante pensar en el cotilleo de la paz, en el rumor de la existencia de hombres que son capaces de sentir, ser tiernos, mostrarse vulnerables, quitarse las armaduras. Que se liberan del macho y regresan al buen vivir.

Para finalizar, los retos que tiene ahora el acuerdo de paz en La Habana son en su implementación debido a la feroz resistencia que han realizado algunos sectores políticos de la extrema derecha colombianos. La visión que nos han dado las activistas y los activistas es que van andando en medio de todas las dificultades que esto implica. Pero, ese camino no podría hacerse, sin el acompañamiento internacional que han ofrecido distintos países al proceso y la ONU. Así mismo, se evidencia cierta preocupación por la falta de políticas públicas urgentes con relación a las masculinidades nuevas, más allá de actividades que no tienen continuación en el tiempo.

\section{BIBLIOGRAFÍAS BIBLIOGRÁFICAS}

Arroyave, J. (2018). Las masculinidades en contextos de guerra-paz en Colombia. Bogotá.

Bermúdez, M. D. M. (2013). Connel y el concepto de masculinidades hegemónicas: Notas críticas desde la obra de Pierre Bourdieu. Revista Estudos Feministas, 21(1), 283-300. https://doi.org/10.1590/S0104-026X2013000100015

Bourdieu, P. (1996). La dominación masculina. Revista de Estudios de Género, La Ventana, 3, 1-95. https://doi.org/10.32870/lv.v0i3.2683

Connell, R. (2013). Hombres, masculinidades y violencia de género. En S. Cruz, (coord.). Vida, Muerte y resistencia en Ciudad Juárez. Una aproximación desde la violencia, el género y la cultura (pp.261-278). Tijuana: Ed. El Colegio de la frontera norte

Connell, R. (2003). Masculinidades. Ciudad de México: Ed.Universidad Nacional Autónoma de México.

Estrada, J. y Jiménez, C. (2019). Seminario CLACSO Proceso de paz e implementación 
final del acuerdo. Disponible https://repositorio.clacso.org/Proceso_de_paz_colombia.html?fbclid=IwAR1s2q nEgNPUTh4mSEhB0QJwxD9qdMIk1uUG2XTJLAqCOW4abG8yzR9T3pE

Méndez, L. B. (2002). Masculinidad hegemónica e identidad masculina. Dossiers feministes, $0(6), 7-35$.

Millán, C., Serrato, L., Pérez, O., Castro, C., Estupiñan, D., y Ruiz, A. (2015). Buenaventura: Un puerto sin comunidad. Bogotá: Centro Nacional de Memoria Histórica

Muñoz, F. A. (2001) (ed). La Paz imperfecta. Granada: Editorial Universidad de Granada.

Muñoz, F. A., Jiménez, J. M. (2009). Historia de una paz imperfecta de género. En $\mathbf{M}^{\mathrm{a}}$. E. Díez y M. Sánchez (coord.) Género y Paz (pp.179-218). Barcelona: Icaria.

Muñoz-Onofre, D.R. (2011). Masculinidades bélicas como tecnología de gobierno en Colombia.. La Manzana, 5(9), 96-107.

Noha, Y. (2014). Sapíens de animales a dioses. Ed Debate. Barcelona.

Rubio, M. (2013). No llores por Tanja, Colombia. Ed Fundación ideas para la paz. Bogotá.

Theidon, K. (2009). Reconstrucción de la masculinidad y reintegración de excombatientes en Colombia. Ed Fundación ideas para la paz. Bogotá.

Tonón, G. (2008.). Reflexiones latinoamericanas sobre investigacion cualitativa. Universidad Nacional de la Matanza. Ed Prometeo. Buenos Aires.

Zuleta, E. (1991). Violencia, Democracia y Derechos Humanos. Ed Hombre Nuevo Editores. Medellín. 